Sharif University of Technology
Scientia Iranica
SCIENTIA
IRAN ICAA

\title{
Brain tissue constitutive material models and the finite element analysis of blast-induced traumatic brain injury
}

\author{
A. Eslaminejad, M. Hosseini-Farid, M. Ziejewski, and G. Karami ${ }^{1, *}$ \\ Department of Mechanical Engineering, North Dakota State University, Fargo, ND 58108-6050, USA.
}

Received 3 December 2017; received in revised form 16 May 2018; accepted 28 July 2018

\section{KEYWORDS}

Blast traumatic brain

injury;

Finite element

modelling;

Brain tissue

constitutive model;

Viscoelastic;

Hyperviscoelastic.

\begin{abstract}
Traumatic Brain Injury (TBI) often occurs due to assaulting loads such as blast on the human head. Finite Elements (FEs) can approximately simulate blast interactions with the human head. An important parameter in the FE modelling procedures is the accuracy of constitutive formulation of the brain tissue. This paper focuses on implementation of three brain tissue constitutive relations to measure and compare the dynamic behaviour of the brain under identical blast loads. For the geometry, a simple spherical head model is employed to monitor the brain tissue response and examine the uncertainties in FE brain tissue constitutive modelling. The brain tissue is constitutively modelled as hyperelastic, viscoelastic, and hyperviscoelastic material types. Intracranial Pressures (ICP), strains, and shear stresses as the dynamic parameters are measured with time. These biomechanical parameters can be compared against the injury thresholds. Our analyses show that although the results of ICPs and strains are close for the three models, shear stresses are considerably different. The study will further provide a new insight into selecting a proper constitutive model of the brain tissue under dynamic conditions.
\end{abstract}

(C) 2018 Sharif University of Technology. All rights reserved.

\section{Introduction}

There are many reported TBI incidents due to highfrequency loads of impact, blast waves, or kinematical motions. Analyzing and understanding the mechanism of TBI will help save many lives across the world. Experimental methods impose moral and technical issues before conducting any research. Many FE studies have been conducted to determine brain responses under dynamic loads to study TBIs [1-10]. Such studies have helped to efficiently develop TBI prediction tools based

1. This article is dedicated to Professor Goodarz Ahmadi, my dear teacher and my colleague.

*. Corresponding author. Tel.: +1701 12315859 E-mail address: g.karami@ndsu.edu (G. Karami).

doi: $10.24200 /$ sci. 2018.20888 on the current injury threshold criteria of kinematics, intracranial pressure (ICP), tissue strain, and tissue shear stress [11].

Mechanical responses of human head brain and skull have been studied using multi-material FE simulations [5,12]. Representative FE head models, which include major components of the head such as brain, skull bones, and cerebrospinal fluid (CSF), have been employed to study the response of the head under different loadings. Although FEs have facilitated the biomechanical modeling of the head, they have limitations in accuracy of the solution. Such limitations come from the complexities of geometries and material properties. The interactions and contact between the head components as well as the fluid-solid-type interactions of CSF with the brain and skull fuel the aforementioned complexities. Thus, in general, we should expect approximate solutions by FE modeling in TBI analysis. 
In a study to be presented in this paper, a spherical model is to be employed to simulate a complicated human head. In FE modeling of such a spherical model, compared to a cadaveric head model, the geometrical complexity will be reduced and, therefore, will have lower computational costs. Furthermore, using such a simple spherical model allows us to focus merely on the effects of material properties instead of being engaged with difficulties and complexities of the human head geometry. Hence, the focus here is to explore the variation of $\mathrm{FE}$ results in TBI, once different tissue constitutive models are implemented.

The intracranial organs and tissues are naturally anisotropic and inhomogeneous. The constitutive models developed for the brain tissue range from linear elastic to nonlinear hyperviscoelastic models. Several experimental studies have shown that the mechanical response of the brain tissue is a function of strain level, strain rate, and load-duration time. Therefore, to predict the biomechanical behavior of the brain, an accurate nonlinear viscoelastic material model of tissue is suggested. Moreover, to consider using such brain tissue constitutive models as utilized in FE simulations [13], their accuracies must be validated against the TBI thresholds, too. Although some tolerances of head and brain injuries have been proposed for the impact-induced TBI, it is still a challenging issue for blast-induced TBIs due to the loading complexity and an unknown injury mechanism.

In the head modeling presented here, skull and brain are modeled as solid elements, while CSF is modeled using fluid-like solid elements. The skull and CSF are modeled as linear elastic materials. It has been proved the brain presents nonlinear elastic and viscoelastic behavioral patterns [13]. Three widely used tissue constitutive models are, therefore, considered for the brain to perform a comparative blast-induced TBI (bTBI) study: hyperelastic, viscoelastic, and hyperviscoelastic models [14]. In related literature, each of these material models claims to represent the behavior of the brain in FE simulation of TBI. Therefore, the aim of this study is to analyze and compare the brain tissue responses using these three models.

\section{Computational method}

\subsection{FE discretization and blast FE modeling}

Three spheres were designed and assembled inside each other to develop a spherical head model. The inner sphere is considered as the brain with a diameter of $D_{\text {brain }}=18.8 \mathrm{~cm}$, which is in contact with the larger sphere as CSF with a diameter of $D_{\mathrm{CSF}}=23.5 \mathrm{~cm}$. The outer sphere is considered as the skull with a diameter of $D_{\text {skull }}=27.26 \mathrm{~cm}$, which is exposed to blast shockwaves. Figure 1(a) and (b) show a schematic of the spherical head model, including the three assembled
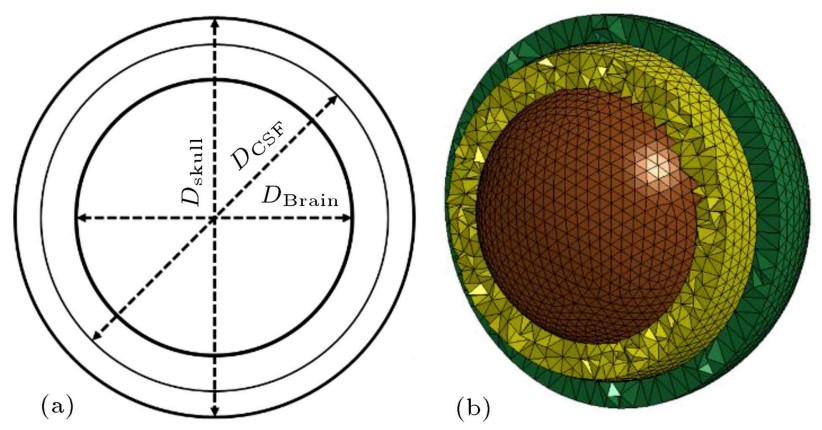

Figure 1. Spherical head model: (a) Three assembled spheres and (b) 3D model and FE discretization.

components, and that of the corresponding FE model, respectively.

To simulate the interaction of blast waves with the spherical head model, the Arbitrary-LagrangianEulerian (ALE) method was implemented. The general numerical algorithm for ALE method is as follows: First, solids deform based on Lagrangian formulations; second, the state variables of the deformed Lagrangian elements are mapped back onto the ALE reference mesh over an advection step; eventually, the governing equations in conjunction with solid relationships are solved for state variables. In addition, the FSI among Lagrangian and Eulerian domains is performed based on penalty method. LS-DYNA explicit, transient, and nonlinear FE code was used to simulate blast shockwaves and their effects on solid bodies.

There are two numerical methods utilized to simulate blast in LS-DYNA:

\section{- (i) Lagrangian Methods (Load-Blast-Enhanced or LBE);}

- (ii) Multi-Material Arbitrary Eulerian-Lagrangian (MM-ALE) [15].

While the first one is unable to simulate shockwave reflections at the edges and is of low accuracy, the second approach operates with huge computation cost. Therefore, the coupled method in LS-DYNA provides a combination of these techniques by taking advantage of both methods to reduce the computation cost and increase accuracy. In this technique, blast overpressure was calculated empirically based on the size and location of the explosive and was applied to the ambient, i.e., the surface facing the detonation (Figure 2(a)). Seventy-gram TNT was considered as the detonation and placed approximately at a standoff distance of $60 \mathrm{~cm}$ from the outer sphere surface. Therefore, the maximum blast overpressure is less than $800 \mathrm{kPa}$ during $0.5 \mathrm{~ms}$. Time history blast overpressure is depicted in Figure 2(b).

For modeling the blast wave, a cubic domain of $50 \mathrm{~cm} \times 50 \mathrm{~cm} \times 50 \mathrm{~cm}$ was constructed and discretized with $6.7 \mathrm{~mm}$ brick elements. In addition, 


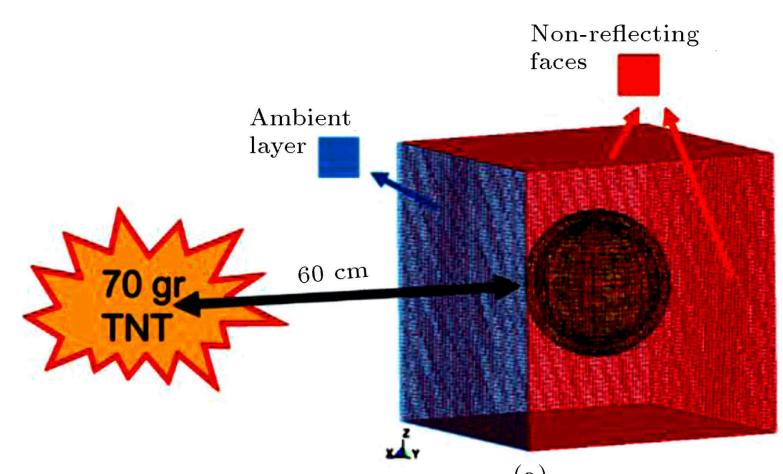

(a)

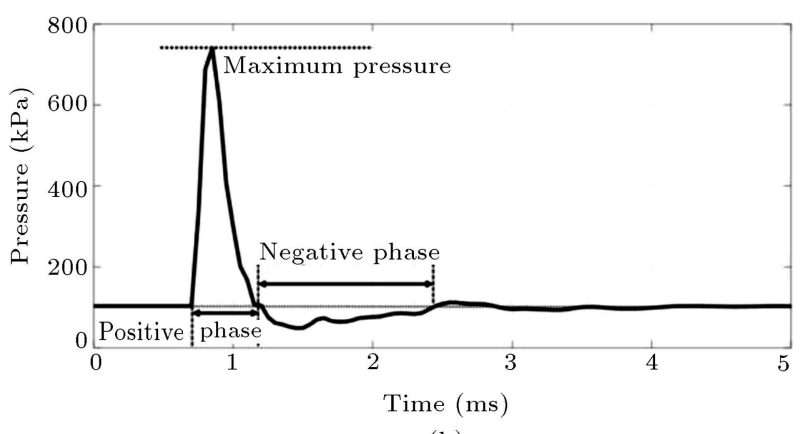

(b)

Figure 2. (a) ALE blast domain and the FE spherical model. (b) Time history of blast overpressure.

the elements placed on the side of the air domain cube in $x$ direction were considered as ambient elements (Figure 2(a)). The blast wave overpressure was applied to these ambient elements. The other sides of the air domain cube were considered as non-reflecting faces in the media-boundary condition. The ambient air was modeled as the ideal gas to model blast wave pressure. Eq. (1) expresses the state of the model, where $p, \gamma, \rho$, and $E$ are pressure, specific heat ratio $\left(\gamma=\frac{C_{p}}{C_{v}}=1.4\right)$, air density, and volumetric energy density, respectively.

$$
p=(\gamma-1) \frac{\rho}{\rho_{0}} E
$$

\subsection{Constitutive models}

The skull was considered as a linear homogeneous and isotropic elastic material. For CSF, the solid elements with fluid properties were used to model it in LSDYNA. The linear elastic properties of the CSF and skull are presented in Table 1, where $\rho$ is density, $E$ is Young's modulus, $v$ is Poisson Ratio, and $K$ is Bulk modulus.
Several studies have focused on determining the brain tissue constitutive models [16,17]. Linear elastic constitutive models have been used in earlier FE studies in order to simplify the brain constitutive model [14]. However, other research efforts have been made on other brain constitutive models: viscoelastic, hyperelastic, and hyperviscoelastic ones [14,18]. A density of $1.04 \mathrm{~g} / \mathrm{cm}^{3}$ was considered for all the brain tissue constitutive models. The adopted mechanical properties of the brain tissue constitutive models are provided in Table 2 in the following sections.

\subsection{Viscoelastic modeling}

Several experimental researches have demonstrated that there is a considerable difference between linear elastic and viscoelastic materials due to loading time, since the brain deformation has viscous modes [14]. Usually, Kelvin viscoelastic model (linear spring and dashpots) is considered for the brain material model. Modeling linear viscoelastic materials in terms of stress-strain relationship need to be done by convolution integrals. Thus, to obtain the stress tensor, the

Table 1. Material properties of CSF and skull.

\begin{tabular}{ccccc}
\hline $\begin{array}{c}\text { Elastic material } \\
\text { properties }\end{array}$ & $\begin{array}{c}\text { Density, } \boldsymbol{\rho} \\
\left(\mathrm{g} / \mathbf{c m}^{\mathbf{3}}\right)\end{array}$ & Poisson ratio, $\boldsymbol{v}$ & $\begin{array}{c}\text { Elastic modulus, } \boldsymbol{E} \\
(\mathbf{G P a})\end{array}$ & $\begin{array}{c}\text { Bulk modulus, } \boldsymbol{K} \\
(\mathbf{G P a})\end{array}$ \\
\hline CSF & 1.04 & 0.4887 & 0.0148 & 2.19 \\
Skull & 1.8 & 0.21 & 15 & - \\
\hline
\end{tabular}

Table 2. Mechanical properties of brain tissue constitutive models.

\begin{tabular}{|c|c|c|c|c|c|c|c|c|}
\hline Material models & \multicolumn{8}{|c|}{ Mechanical properties } \\
\hline \multirow{2}{*}{ Hyperelastic } & Density $\left(\mathrm{g} / \mathrm{cm}^{3}\right)$ & Poisson ratio & $C_{10}(\mathrm{~Pa})$ & & & $C_{01}(\mathrm{~Pa})$ & & \\
\hline & 1.04 & 0.499994 & 514.62 & & & 566.08 & & \\
\hline \multirow{2}{*}{ Viscoelastic } & Density $\left(\mathrm{g} / \mathrm{cm}^{3}\right)$ & Bulk modulus ( $\mathrm{GPa}$ ) & $G_{0}(\mathrm{kPa})$ & & $G_{\infty}(\mathrm{kPa})$ & & $\beta(1 / \mathrm{sec})$ & \\
\hline & 1.04 & 2.19 & 43 & & 8 & & 500 & \\
\hline \multirow{2}{*}{ Hyperviscoelastic } & Density $\left(\mathrm{g} / \mathrm{cm}^{3}\right)$ & Bulk modulus (GPa) & $C_{10}(\mathrm{~Pa})$ & $C_{01}(\mathrm{~Pa})$ & $G_{1}(\mathrm{kPa})$ & $G_{2}(\mathrm{kPa})$ & $\beta_{1}(\mathrm{~Hz})$ & $\beta_{2}(\mathrm{~Hz})$ \\
\hline & 1.04 & 2.19 & 3102.5 & 3447.2 & 40.74 & 23.285 & 125 & 6.67 \\
\hline
\end{tabular}




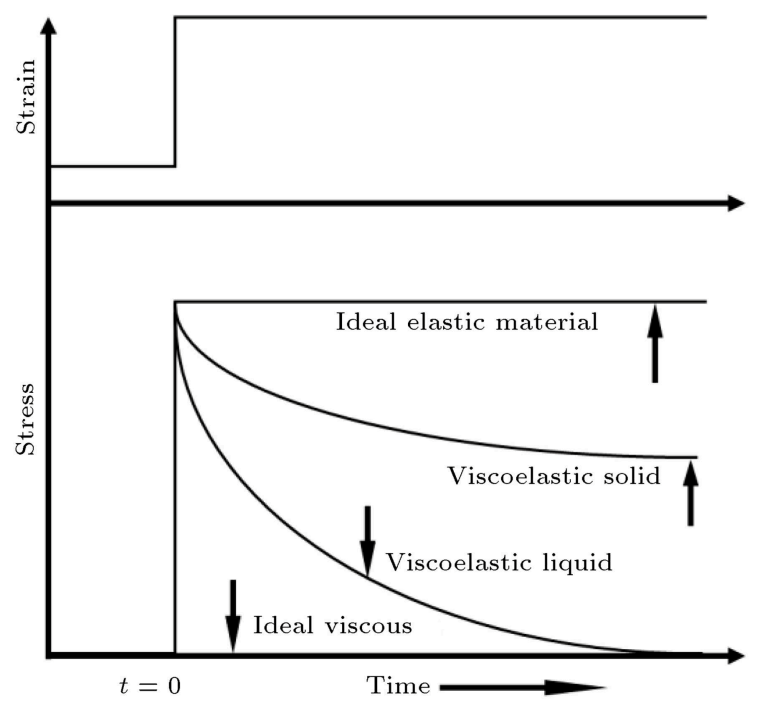

Figure 3. Applied strain and the stress relaxation responses with time of tissue material.

convolution integral is written in Eq. (2). It should be noted that the compressible manner of the brain is considered as a linear behavior.

$$
\dot{S}_{i j}=2 \int_{0}^{t} \phi(t-\tau) \frac{\partial \varepsilon_{i j}(\tau)}{\partial \tau} d \tau,
$$

where $\dot{S}_{i j}$ is the Piola-Kirchhoff stress rate. In addition, the shear relaxation modulus $(\phi)$ is obtained through Eq. (3):

$$
\phi(t)=G_{\infty}+\left(G_{0}+G_{\infty}\right) e^{-\beta t},
$$

where $G_{\infty}$ is the long-term shear modulus, $G_{0}$ is the short-term shear modulus, and $\beta$ is the decay factor. These three unknown coefficients can be obtained by stress relaxation response with time subjected to strain with constant strain rate. As is presented in Figure 3, the stress-time graph can obtain the unknown coefficients of the shear relaxation modulus formula. Viscoelastic material properties used in our study based on cadaveric tests are provided in Table 2 [14].

\subsection{Hyperelastic modeling}

Some researchers have considered hyperelastic properties of the brain tissue and introduced them to FE modeling of brain. The strain energy function (Mooney-Rivlin) is modeled as a polynomial function of the principal strain invariant to cope with high-rate elastic deformation as follows:

$$
W=\sum_{i+j=1}^{N} C_{i j}\left(J_{1}-3\right)^{i}\left(J_{2}-3\right)^{j},
$$

where $J_{1}, J_{2}$, and $J_{3}$ can be obtained by:

$$
\begin{aligned}
& J_{1}=\operatorname{Trace} \mathbf{B}, \\
& J_{2}=\frac{J_{1}-\operatorname{Trace}^{2}}{2 J_{3}}, \\
& J_{3}=\sqrt{\operatorname{det} \mathbf{B}}=1,
\end{aligned}
$$

where $\mathbf{B}$ is a left Cauchy-Green strain tensor. $\lambda$ is obtained by using the tensile experimental data [16].

$$
\mathbf{B}=\left[\begin{array}{ccc}
\lambda_{z}^{2} & 0 & 0 \\
0 & \lambda_{z}^{-1} & 0 \\
0 & 0 & \lambda_{z}^{-1}
\end{array}\right]
$$

By considering the first two terms of the strain energy, the Mooney-Rivlin strain energy function for incompressible materials [18] is written as follows:

$$
W=C_{10}\left(I_{1}-3\right)+C_{01}\left(I_{2}-3\right),
$$

where $I_{1}$ and $I_{2}$ are the first and second principal stress invariants, respectively. The sum of $C_{10}$ and $C_{01}$ has a physical meaning as shear modulus $\mu_{0}$ :

$$
\frac{1}{2} \mu_{0}=C_{10}+C_{01}
$$

where, in this study, $\mu_{0}$ is shear modulus about $2160 \mathrm{~Pa}$. As one of the assumptions, the relation between constants is $\frac{C_{10}}{C_{01}}=0.9$. In addition, the bulk modulus is obtained as $2.19 \mathrm{GPa}$ for the calculated Poisson ratio [18]. The Cauchy Stress is obtained using the principal Kirchhoff stress components from derivative of strain energy function:

$$
\sigma_{i j}=J^{-1} F_{i k} S_{k m} F_{m j}^{T},
$$

where $J, F$, and $S$ are Jacobian transformation, deformation gradient tensor, and Second Piola-Kirchhoff stress, respectively, which can be obtained by Eq. (10). Based on the experimental data fitting, the coefficients were estimated to use the Mooney-Rivlin hyperelastic model [18]. Moreover, mechanical property coefficients of the hyperelastic constitutive model are provided in Table 2.

$$
S_{i i}=\lambda_{i} \frac{\partial W}{\partial \lambda_{i}}
$$

\subsection{Hyperviscoelastic modeling}

The Mooney-Rivlin strain energy function is considered as the third constitutive model studied in this paper. The time decay constant and time-dependent constants of Mooney-Rivlin were derived in terms of time by Mendis et al. [17]. Indeed, viscous dissipative effects were taken into viscous stress, which was related to the elastic stress. Hence, the second Piola-Krichhoff stress can be obtained by utilizing the convolution integral (Eq. (11)) [1]:

$$
S_{i j}=\int_{0}^{t} G_{i j k l}(t-\tau) \frac{\partial E_{k l}}{\partial \tau} d \tau,
$$

where $E_{k l}$ is the Green's strain, and $G_{i j k l}$ is the stress 
relaxation function:

$$
G(t)=\sum_{i=1}^{n} G_{i} e^{-\beta_{i} t},
$$

where $0.5 G_{i}=\left(C_{01}+C_{10}\right)$. Time-dependent coefficients $C_{10}$ and $C_{01}$ for Mooney-Rivlin hyperelastic law are obtained as follows:

$$
C_{01}(t)=A C_{10}(t)=a+b e^{\frac{-t}{c}}+d e^{\frac{-t}{c}} .
$$

Hereby, the Cauchy Stress can be written for a hyperviscoelastic model in Eq. (13), in which $\mathbf{F}$ is the deformation gradient tensor, $\mathbf{J}$ is the transformer Jacobian, and $\mathbf{S}$ is the second Piola-Krichhoff stress.

$$
\sigma=\mathbf{J} \cdot \mathbf{F}^{T} \cdot \mathbf{S} \cdot \mathbf{F}
$$

The mechanical property coefficient can be obtained by utilizing mechanical tests such as tensile and compression tests on brain tissue. Hyperviscoelastic material properties used in our study are provided in Table 2. Hyperviscoelastic constitutive brain tissue model has been used by researchers [19] who have validated their results by experimental tests in terms of ICP responses.

\section{Result and discussions}

In this study, three material models (hyperelastic, viscoelastic, and hyperviscoelastic) were utilized to model brain tissue under high-frequency blast loading. The blast pressure wave propagation is depicted in Figure 4. At the time when the blast pressure wave reached the outer sphere, the maximum pressure was evaluated as about $780 \mathrm{kPa}$. In addition, the outer sphere (skull) moves with maximum acceleration about

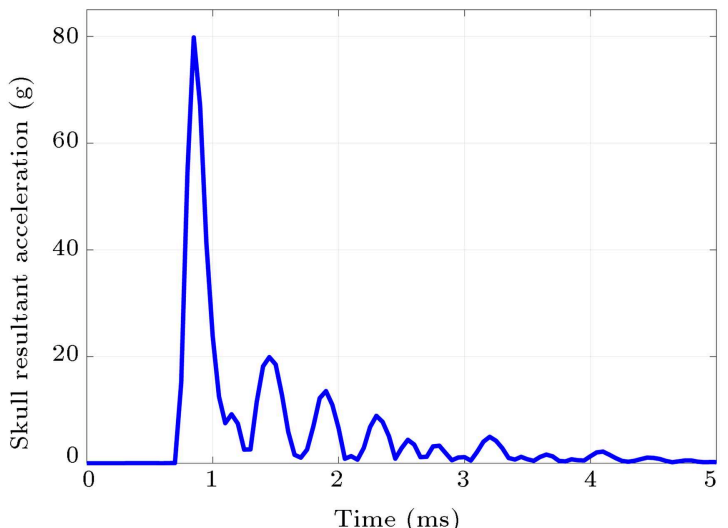

Figure 5. Skull acceleration of hyperviscoelastic model due to the blast.

$80 \mathrm{~g}$ (Figure 5). This amount of blast pressure was selected to produce the skull acceleration close enough to the threshold of mild TBI [11].

To quantify the brain injury levels, several criteria have been proposed as found in the literature: acceleration, ICP, strain, and stress (mostly shear). Of note, mostly, in engineering application, the failure at a point is measured based on the size of the stress. However, in biomechanics and brain injury studies, for the convenience and following reasons, other failure criteria have been introduced:

- Acceleration can be easily measured from experiments of the dummy heads (Hybrid III) models;

- ICP can be compared with the result of cadaver head models test;

- The size of strain involves either in-vitro compression or tension tests of brain tissue.

Any of the mentioned criteria used to measure the intracranial brain deformation leads to tissue damage.

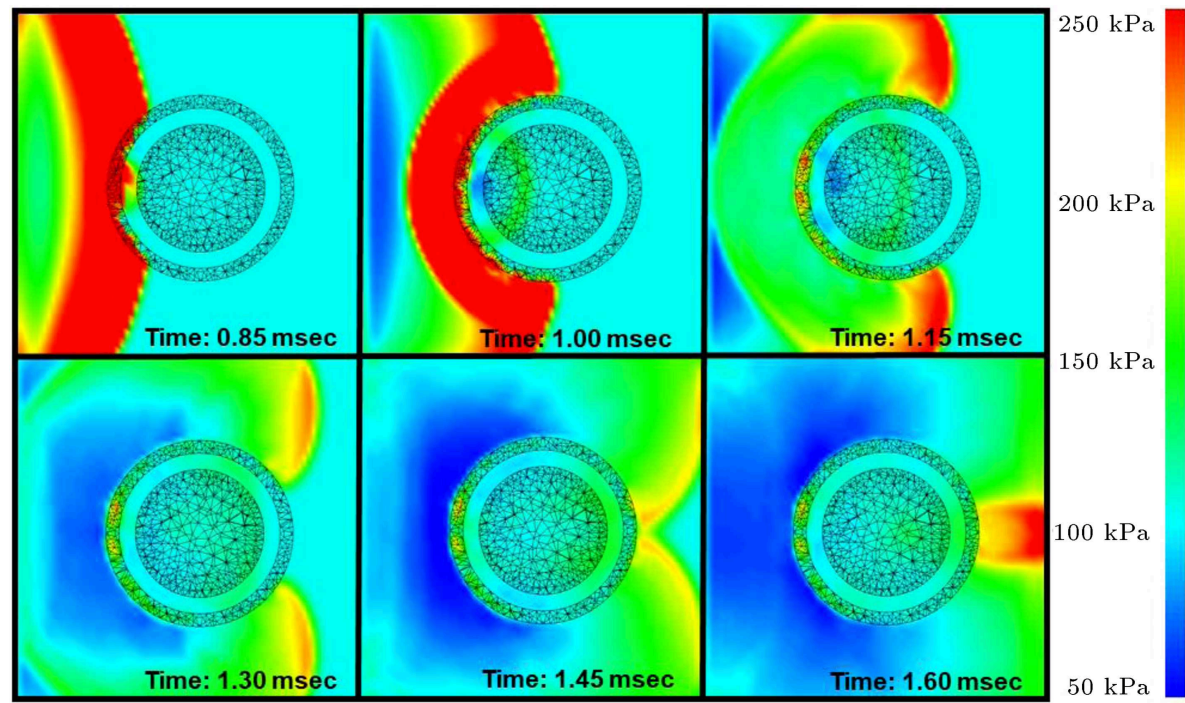

Figure 4. Blast wave pressure interactions with spherical head model. 


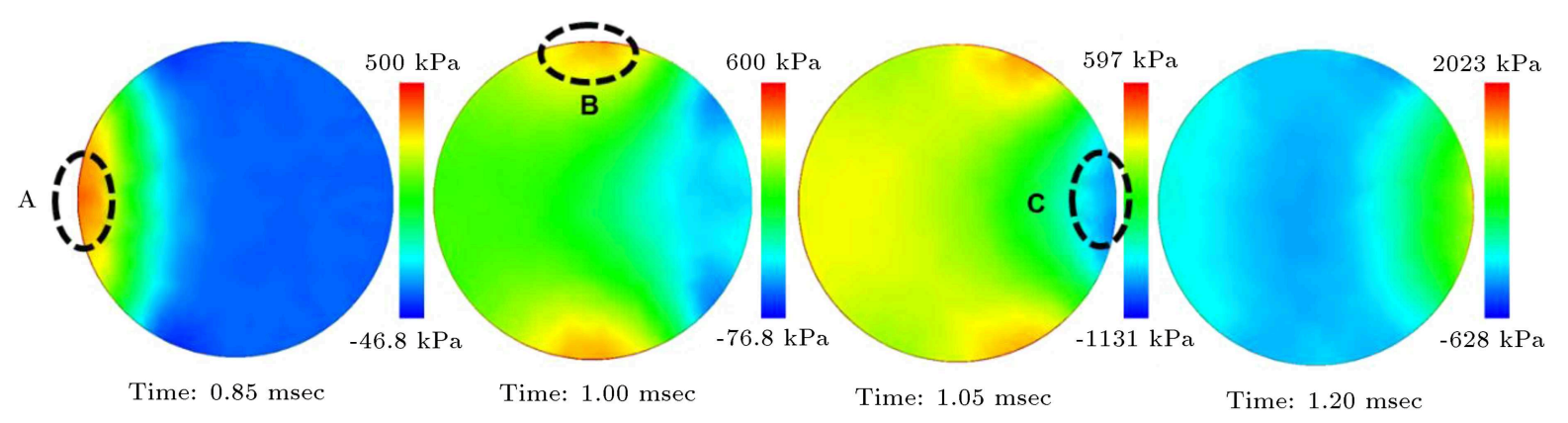

Figure 6. Pressure wave propagation in the inner sphere.

Eventually, the size of the shear stress causes fiber distortion, shear, rupture, and failure for the brain tissue. FE analysis can effectively measure the size of shear stresses in any type of analysis.

The propagated pressure wave inside the inner sphere (brain) is demonstrated in Figure 6. As seen at $t=1 \mathrm{~ms}$, a small pressure wave starts to propagate inside the skull, CSF, and brain media. As is indicated in Figure 6, there are three regions (regions $\mathrm{A}, \mathrm{B}$, and $\mathrm{C}$ ), in which pressure concentration is considerable. These regions experience considerable pressure in an inner sphere marked as Locations A, $\mathrm{B}$, and $\mathrm{C}$ that are located in the front (coup), top site, and backside (contrecoup) regions, respectively. For the three tissue material models, the ICPs were measured and recorded. While hyperviscoelastic and viscoelastic materials predicted a similar maximum ICP peak about $500 \mathrm{kPa}$ in the coup site (Location A), the hyperelastic model predicted an overpressure of $420 \mathrm{kPa}$. In addition, the behavior of these models was considerably different in terms of damping and settling down. The hyperelastic model predicts faster damping than the other two models do.

Another pressure concentration region was detected at top of the brain sphere in Location B. In hyperviscoelastic and viscoelastic models, the maximum ICP responses in the top region were close to about $600 \mathrm{kPa}$ and $591 \mathrm{kPa}$, respectively. However, for the hyperelastic model, the ICP response was $445 \mathrm{kPa}$. Time histories of ICP variation in Locations A, B, and $\mathrm{C}$ are depicted in Figure 7 . It can be seen that while hyperviscoelastic and viscoelastic models have very similar Root Mean Square (RMS) values of 136.47 and 136.68, the hyperelastic model has an RMS value of 81.06 , which is considerably less than RMS values of the other two models.

As shown in Figure 7, the pressure response in countercoup (Location C) was found significantly higher than those at other regions with peak rates about $2.02 \mathrm{MPa}, 2.04 \mathrm{MPa}$, and $2.11 \mathrm{MPa}$ for hyperviscoelastic, viscoelastic, and hyperelastic models, respectively. Although the maximum ICP occurred in the hyperelastic model, the RMS values of the
ICPs in hyperviscoelastic (310.94) and viscoelastic models (313.61) were more than that of hyperelastic model (258.31). The pressure propagation contour is demonstrated in Figure 6 that illustrates the maximum pressure occurring in Location $\mathrm{C}$ on the outer surface of the brain and not deep through the brain tissue.

It is observed that at point $\mathrm{C}$ on the brain (inner sphere), ICP varied significantly, which can be due to blast pressure concentration at countercoup. The maximum pressure at point $\mathrm{C}$ was approximately four times larger than pressure in Locations $\mathrm{A}$ and $\mathrm{B}$. In addition, the considerable negative pressure, about $-1.5 \mathrm{MPa}$, in countercoup (Location $\mathrm{C}$ ) can anticipate the cavitation phenomenon.

Time histories of the maximum strain response in all Locations A, B, and C are depicted in Figure 8. Unlike the hyperelastic model, the maximum principal strains of the viscoelastic and hyperviscoelastic models in Locations A and B illustrate similar responses under blast. The dynamic response of the models under the blast shockwave showed approximately a similar strain response with RMS values of $0.0165 \%, 0.0170 \%$, and $0.0166 \%$ for hyperviscoelastic, viscoelastic, and hyperelastic models, respectively, in the countercoup location.

Finally, the maximum shear stress responses to the blast are calculated that show significant differences, as depicted in Figure 9. The shear stress criterion is important because it is the main cause of tissue layer distortion and nervous fiber fracture, resulting in diffuse injuries. The maximum shear stresses at point A for hyperviscoelastic, viscoelastic, and hyperelastic material models were $0.14 \mathrm{kPa}, 0.34 \mathrm{kPa}$, and $0.20 \mathrm{kPa}$, respectively. However, in Location $\mathrm{C}$, the hyperelastic material model had the maximum shear stress about $1.37 \mathrm{kPa}$, which was larger than viscoelastic and hyperviscoelastic models' maximum shear stresses of $1.12 \mathrm{kPa}$ and $0.395 \mathrm{kPa}$. Since the shear stress in the regions is noisy, the Savitzky-Golay Filter was applied to smooth the measured data.

The maximum shear stress was much larger in the countercoup of the brain sphere in comparison with coup, which was facing blast wave load. The 


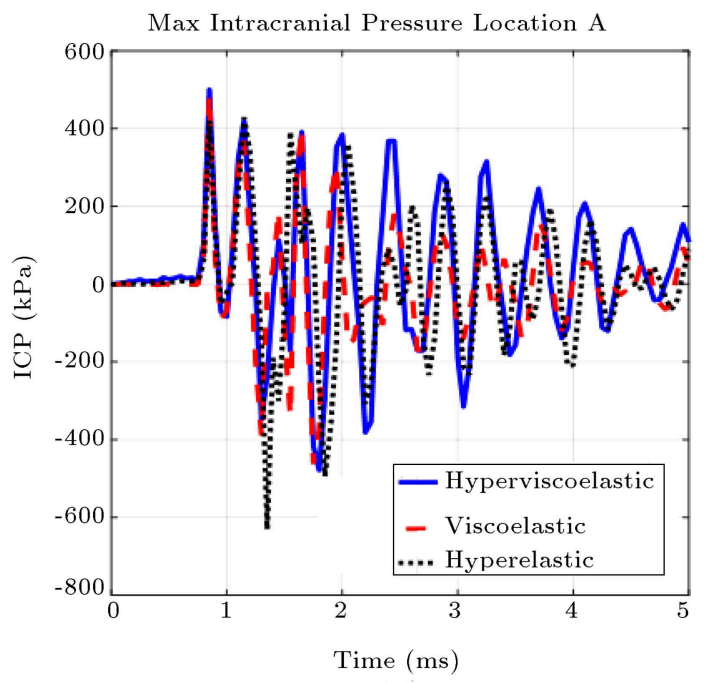

(a)

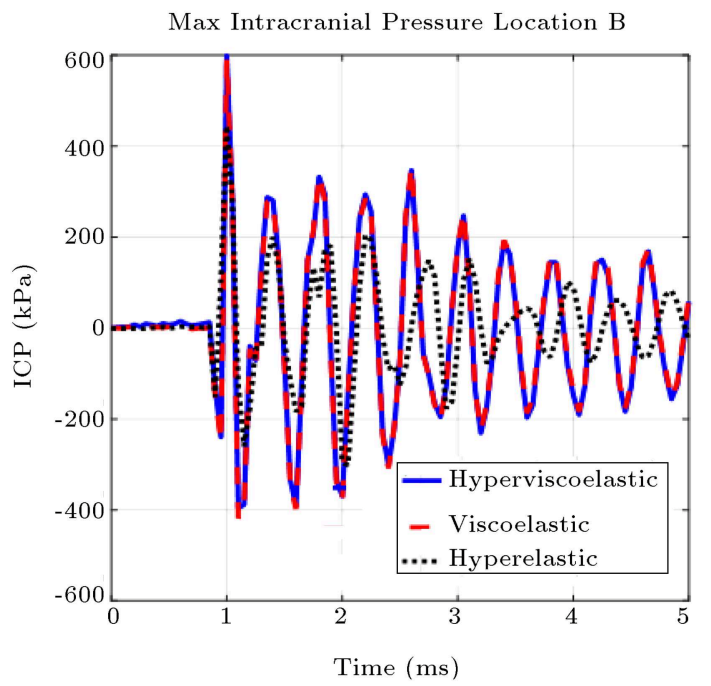

(b)

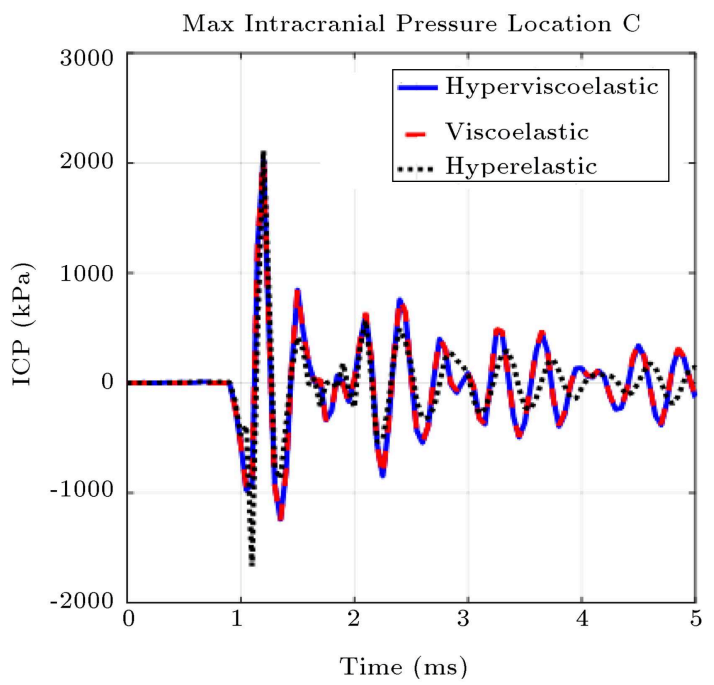

(c)

Figure 7. ICP variation with time at front (a), top (b), and backside (c) of the inner sphere.

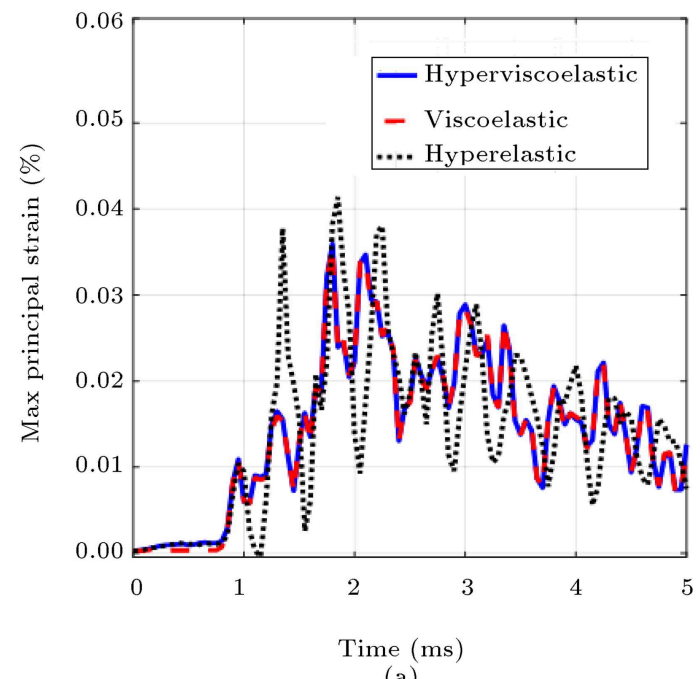

(a)

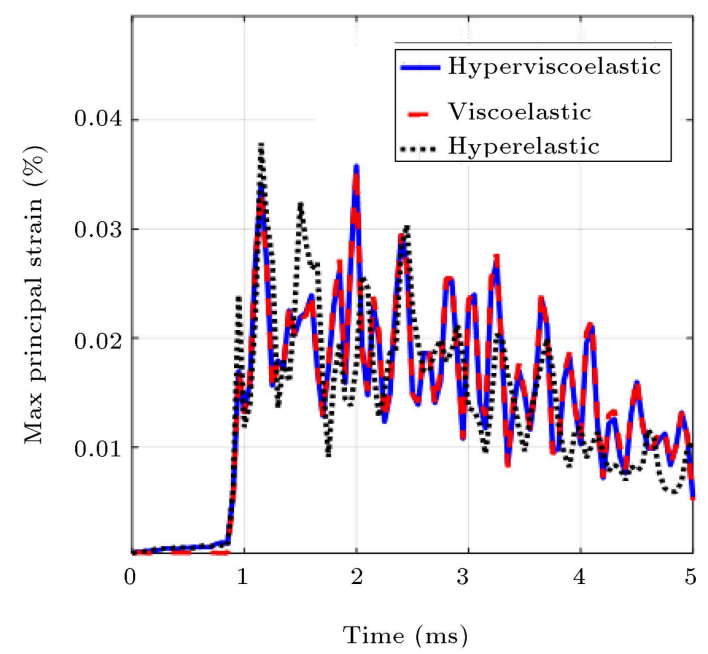

(b)

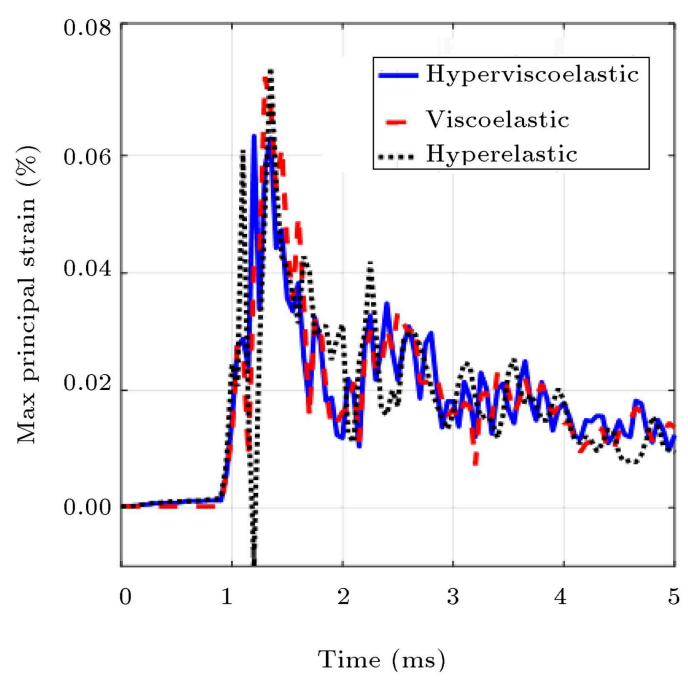

(c)

Figure 8. Maximum principal strain in: (a) Location A, (b) Location B, and (c) Location C. 


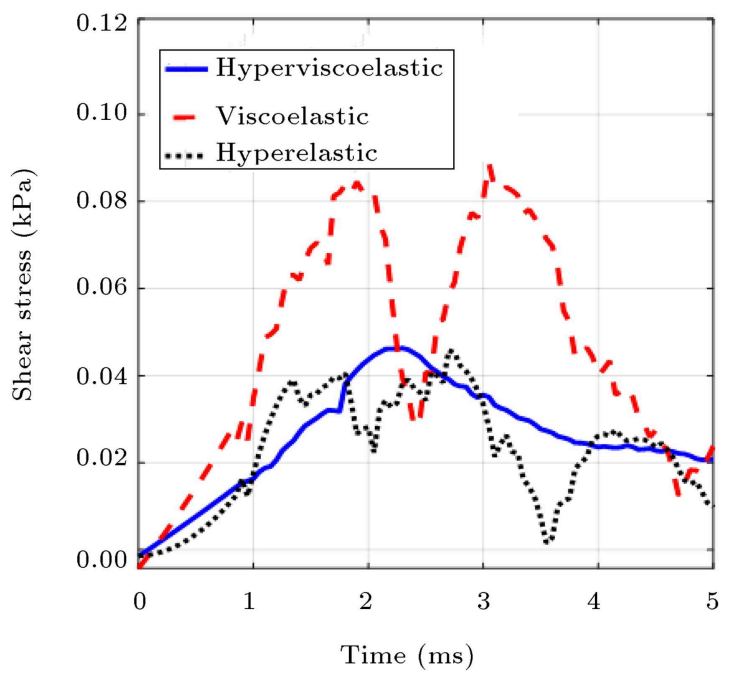

(a)

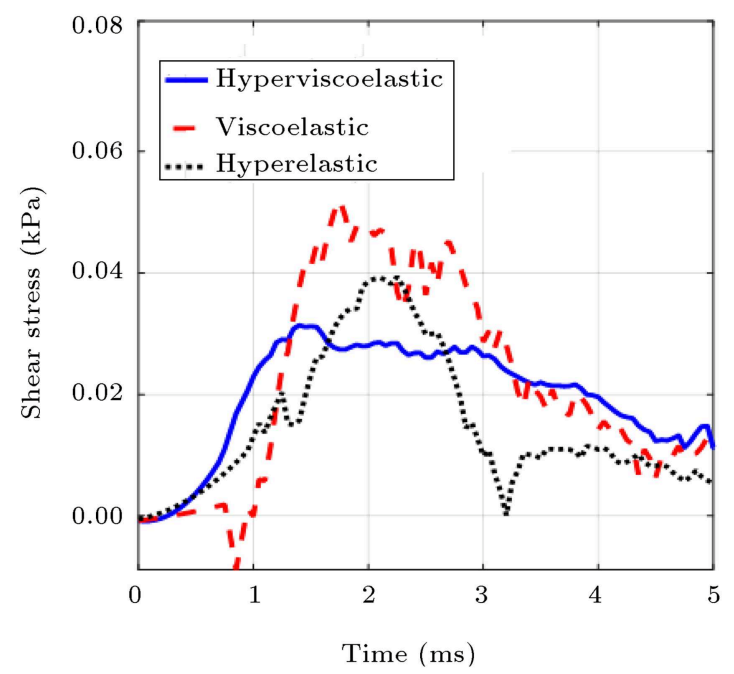

(b)

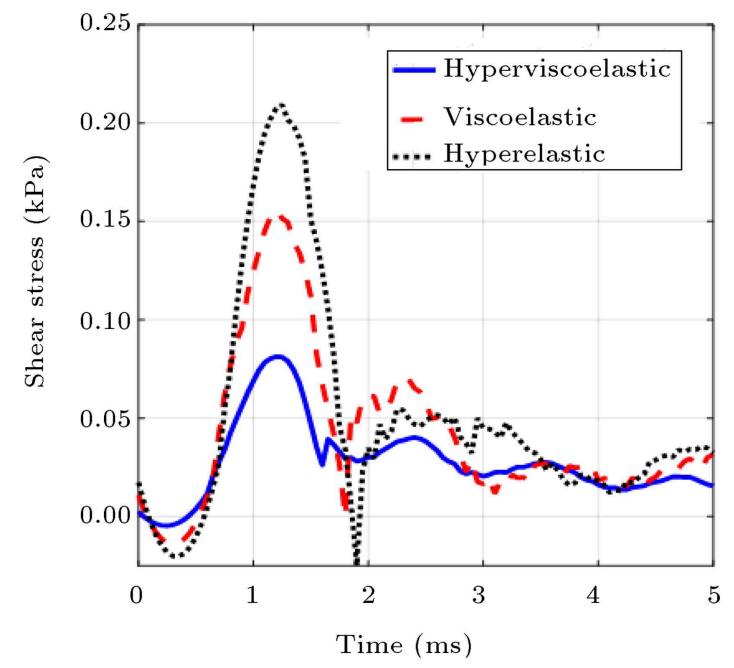

(c)

Figure 9. Maximum shear stress in (a) Locations A, (b) Location B, and (c) Location C. viscoelastic brain model had the maximum shear peak in comparison with hyperviscoelastic and hyperelastic models in coup and countercoup sites. The bulk modulus of the brain is high enough to present the incompressibility in the tissue. Thus, it should be noticed that ICPs in the brain, which is generated by the mean stress (hydrostatic pressure), are found to be similar for all three material models. However, the deviatoric stresses, which are in charge of the shear stresses and tissue distortions, were recognized to present different responses in each material model.

\section{Conclusion}

Three material models, namely hyperviscoelastic, viscoelastic, and hyperelastic, were considered as brain tissue models to study the dynamic responses under blast shockwaves. The head model was simplified into a sphere shape to overlook directionality, decrease computation costs, and reduce the complexity of the geometry. Skull, CSF, and brain were considered as three interconnected sphere models.

The tissue dynamic responses were studied in terms of maximum ICP, maximum principal strain, and maximum shear stress. The limitations of these parameters stand as the threshold criteria for TBI. These dynamic values for each of the constitutive models were monitored in the coup and countercoup regions, where their maximum values occur. The countercoup region had the highest ICP, especially on the outer surface of the brain, approximately four times larger than that at the front and top of the spherical brain. The hyperviscoelastic and viscoelastic models had close ICP values; however, for the hyperelastic model, the maximum ICP was lower. The negative pressures, which might claim the cavitation on brain under blast in all cases, occur in the coup and countercoup regions. Maximum principal strains' changes with time for the viscoelastic and hyperviscoelastic models are similar to ICP's changes with time. Generally, the three constitutive models present a similar response of ICP and strain; specifically, the results of the two models (viscoelastic and hyperviscoelastic) were close to each other. The shear stresses of all the models behave differently and predict different responses. Such differences necessitate regarding the selection of the type of constitutive model as highly important. In addition, as the shear stress is the essential criterion for material failure, it must be considered and compared to the shear stress threshold for TBI.

\section{References}

1. Chafi, M., Karami, G., and Ziejewski, M. "Biomechan- 
ical assessment of brain dynamic responses due to blast pressure waves", Annals of Biomedical Engineering, 38(2), pp. 490-504 (2010).

2. Cotton, R., Pearce, C.W., Young, P.G., Kota, N., Leung, A., Bagchi, A., and Qidwai, S. "Development of a geometrically accurate and adaptable finite element head model for impact simulation: the Naval research laboratory-impleware head model", Computer Methods in Biomechanics and Biomedical Engineering, 19(1), pp. 101-113 (2016).

3. Eslaminejad, A., Sarvghad-Moghaddam, H., Rezaei, A., Ziejewski, M., and Karami, G. "Comparison of brain tissue material finite element models based on threshold for traumatic brain injury", ASME 2016 International Mechanical Engineering Congress and Exposition, Phoenix, AZ, USA: American Society of Mechanical Engineers (2016).

4. Sarvghad-Moghaddam, H., Rezaei, A., Eslaminejad, A., Ziejewski, M., and Karami, G. "Mechanical response of the brain under blast: The effect of blast direction and the head protection", ASME 2016 International Mechanical Engineering Congress and Exposition. Phoenix, AZ, USA: American Society of Mechanical Engineers (2016).

5. Sarvghad-Moghaddam, H., Rezaei, A., Ziejewski, M., and Karami, G. "Evaluation of brain tissue responses due to the underwash overpressure of helmet and faceshield under blast loading", International Journal for Numerical Methods in Biomedical Engineering, 33(1), Electrical article: E02782 (2016).

6. Grujicic, M., Bell, W.C., Pandurangan, B., and He, T. "Blast-wave impact-mitigation capability of polyurea when used as helmet suspension-pad material", Materials \& Design, 31(9), pp. 4050-4065 (2010).

7. Moore, D.F., Jérusalem, A., Nyein, M., Noels, L., Jaffee, M.S., and Radovitzky, R.A. "Computational biology-modeling of primary blast effects on the central nervous system", Neuroimage, 47, pp. T10-T20 (2009).

8. Nyein, M.K., Jason, A.M., Yu, L., Pita, C.M., Joannopoulos, J.D., Moore, D.F., and Radovitzky, R.A. "In silico investigation of intracranial blast mitigation with relevance to military traumatic brain injury", Proceedings of the National Academy of Sciences (2010).

9. Sarvghad-Moghaddam, H., Rezaei, A., Ziejewski, M., and Karami, G. "A comparative study on the protection efficiency of combat helmets against ballistic impacts and blast waves", in Journal of Head Trauma Rehabiliation, 12th Annual Conference on Brain Injury, North American Brain Injury Society, San Antonio, Texas, April 29-May 2, pp. E66-E66 (2015).

10. Sarvghad-Moghaddam, H., Karami, G., and Ziejewski, M. "The effects of directionality of blunt im- pacts on mechanical response of the brain", ASME International Mechanical Engineering Congress and Exposition, Montreal, Canada: American Society of Mechanical Engineers (2014).

11. Zhang, L., Yang, K.H., and King, A.I. "A proposed injury threshold for mild traumatic brain injury", Journal of Biomechanical Engineering, 126(2), pp. 226-236 (2004).

12. Sarvghad-Moghaddam, H., Jazi, M.S., Rezaei, A., Karami, G., and Ziejewski, M. "Examination of the protective roles of helmet/faceshield and directionality for human head under blast waves", Computer Methods in Biomechanics and Biomedical Engineering, 18(16), pp. 1846-1855 (2015).

13. Hoseini-Farid, M., Eslaminejad, A., Ziejewski, M., and Karami, G. "A study on the effects of strain rates on characteristics of brain tissue", ASME International Mechanical Engineering Congress and Exposition, Tampa, FL, USA: American Society of Mechanical Engineers (2017).

14. Tse, K.M., Lim, S.P., Tan, V.B.C., and Lee, H.P. "A review of head injury and finite element head models", American Journal of Engineering, Technology and Society, 1(5), pp. 28-52 (2015).

15. "LS-DYNA, LS-DYNA keyword user manual", Version 971, Livermore Software Technology Corporation: Livermore, California (2007)

16. Miller, K. and Chinzei, K. "Constitutive modelling of brain tissue: experiment and theory", Journal of Biomechanics, 30(11), pp. 1115-1121 (1997).

17. Mendis, K., Stalnaker, R., and Advani, S. "A constitutive relationship for large deformation finite element modeling of brain tissue", Journal of Biomechanical Engineering, 117(3), pp. 279-285 (1995).

18. Chafi, M.S., Ganpule, S., Gu, L., and Chandra, N. "Dynamic response of brain subjected to blast loadings: influence of frequency ranges", International Journal of Applied Mechanics, 3(04) pp. 803-823 (2011).

19. Sarvghad-Moghaddam, H. "Computational biomechanics of blast-induced traumatic brain injury", Role of Loading Directionality, Head Protection, and Blast Flow Mechanics, North Dakota State University, Ph.D. Thesis (2015).

\section{Biographies}

Ashkan Eslaminejad is a $\mathrm{PhD}$ Candidate in Mechanical Engineering program at North Dakota State University (NDSU). He received Master of Engineering in Mechanical Engineering from University of Malaysia in Kuala Lumpur, Malaysia. He joined the Computational Biomechanics Lab at NDSU in Fall 2015. His research areas of interest include experimental and theoretical vibration analysis, computational mechanics of fluid-structure interaction systems, and biomechanics 
of human head.

Mohammad Hosseini Farid is a PhD Candidate at the Mechanical Engineering Department at North Dakota State University (NDSU). He received his Master's degree in Mechanical Engineering from Iran University of Science and Technology. His major research thrust is in the area of tissue engineering of the brain and study of the biomechanics of the trauma and impact.

Mariusz Ziejewski is a Professor at the Department of Mechanical Engineering at the North Dakota State University. He received his PhD in Mechanical Engineering in 1986 from North Dakota State
University. The areas of research interests of Dr. Ziejewski include biomechanics of the trauma and impact, head/neck/brain computer modeling, and vehicle dynamics.

Ghodrat Karami is a Professor and the Graduate Program Coordinator at the Mechanical Engineering Department at North Dakota State University (NDSU). He received his Master's degree (1980) and $\mathrm{PhD}$ (1984) in Mechanical Engineering from Imperial College of Science and Technology, University of London, England. Dr. Karami's research interests include multi-scale computational biomechanics, tissue engineering, constitutive modeling, composite engineering, and engineering design. 